\title{
Drug distribution management system based on IOT
}

\author{
Zeliang Liu ${ }^{1,2, *}$, Chunmei Zhang ${ }^{3}$, Hui Peng ${ }^{1}$, Qin Xu' ${ }^{1}$, and Yubao Gao ${ }^{1}$ \\ ${ }^{1}$ School of Electronic and Information Engineering, Jiujiang University \\ Jiujiang, Jiangxi, 332005, China \\ [e-mail: aptblaze@163.com, penghui@jju.edu.cn, 249768604@qq.com, ybgao_jju@163.com] \\ 2 Jiangxi Provincial Key Laboratory for Industrial Ecological Simulation and Environmental Health in Yangtze \\ River Basin, Jiujiang University \\ Jiujiang, Jiangxi, 332005, China \\ [e-mail: aptblaze@163.com] \\ ${ }^{3}$ College of Air and Missile Defense, Air Force Engineering University \\ Xi'an, Shanxi, 710051, China \\ [e-mail: ziyoudehuxi2015@sina.com] \\ *Corresponding author: Zeliang Liu
}

Received August 10, 2021; revised December 24, 2021; accepted January 30, 2022;

published February 28, 2022

\begin{abstract}
In hospitals and pharmacies, the distribution of medicines is an important part. Any mistakes, misses, fake medicines and expired medicines can cause medical accidents. With the widespread application of the Internet of Things technology (IoT), traditional drug distribution methods need to be upgraded. This article proposes a drug distribution management scheme based on the Internet of Things technology. In the production of drugs, a flexible RFID tag was printed on the packaging box, which stored a series of information such as drug name, dosage, raw materials, efficacy, production date, expiration date, and manufacturer. The use of a drug distribution management system combined with RFID readers can identify drug information and effectively prevent the occurrence of erroneous, missed, counterfeit, and expired drugs. It can also improve management efficiency, reduce management costs, and control management risks. Through the circuit design and software system development, the test results show that this solution is effective and feasible, the proposed method can achieve the expected results.
\end{abstract}

Keywords: Internet of things, RFID, drug management system, EPC

This work was financially supported by Science and Technology Project of Jiangxi Provincial Department of Education, China (GJJ201802, GJJ161083). 


\section{Introduction}

In 2016, there were 4176 raw material drug and preparation manufacturing companies in China, at the same time 4011 new drug clinical trials were approved and 49000 drug complaints were accepted [1]. In 2017 and 2018, these data were 4376, 734, 58000 and 4441, 312, 64000, respectively [2,3]. It can be seen from the National Medical Products Administration's "Annual Report on Drug Supervision Statistics" in the past three years that drug manufacturers have continued to increase, but the approval of new drugs has slowed down, and the number of drug complaints has increased. This is largely due to the inability of our drug management programs to keep up with the times. On one hand, the social demand for drugs is increasing, and on the other hand, effective methods for drug supervision are lacking. In August 2019, the State Administration of Drug Administration issued a three-information standard announcement such as the "Basic Technical Requirements for Drug Traceability System", which is applicable to regulate drug market license holders, manufacturing enterprises, operating enterprises, disease prevention, and control institutions, and users Participants in drug information traceability systems such as third-party technical institutions and other parties build and use drug traceability systems [4]. This puts forward higher requirements for the investigation and punishment of counterfeit and inferior medicines and expired medicines, which meets the needs of society.

Drug distribution management is an important part of the state's standardized management, monitoring, and supervision of drugs. Traditional drug distribution management methods are generally the following two types, one is to take the medicine according to the customer's needs or the prescription issued by the doctor, and manually identify the drug packaging, name, model, quantity, expiration date, etc. This method is more traditional, requires a lot of human resources, and simply relies on personnel for identification, which is relatively inefficient.

With the gradual improvement of Chinese residents' health care awareness, major hospitals are overcrowded. Hospitals and pharmacies need to own sufficient service capabilities to meet residents' medical needs. This manual medicine storage and retrieval management method is not only inefficient but also consumes a lot of human resources, which increases the service cost of hospitals and pharmacies. At the same time, with the aging of China's population, it is expected that the medical workload in the next few decades will exceed the service capacity of physicians. Therefore, it is particularly necessary to improve the efficiency of physicians and reduce unnecessary workload. Today, the demand for medicines and the types of medicines continue to expand. In the past, pharmacy management methods, such as manual medicine taking, have gradually failed to meet the demand for rapid drug delivery. With the rapid development of information technology and electronic technology, a second and better solution has emerged.

The second solution is to scan the barcode on the drug package box with a scanner for semi-manual identification, which is a widely used method at present. Later, some researchers also designed a robotic transmission device. Through the OCR recognition system installed on the robot, it can recognize the barcode or two-dimensional code on the drug package and take the medicine according to the requirements of the background data. Although scholars made further use of information technology and made great breakthroughs in drug classification and drug management, the shortcomings of the barcode recognition technology in terms of efficiency, reliability, and anti-counterfeiting cannot meet current social needs. Some scholars have designed and remodeled medicine cabinets using PLCs and motors to improve the 
efficiency of medication dispensing. However, the lack of supervision of medicine management also cannot meet the current social needs.

Of course, these schemes have significantly improved the efficiency compared with the first scheme, but they are not perfect for automation and information management, especially for imitation and traceability. RFID technology can better realize the counterfeit and traceability of medicines. It is an important application branch of the Internet of Things (IoT) technology. With the development of the IoT technology, better solutions have emerged to solve the current dilemma by replacing the above two solutions. IoT-based applications and systems have transformed the world into an imaginary world which human of the 90's thought about and enable the doctors and hospital staff to do their work more precisely and actively with less effort and intelligence [5].

The remainder of this paper is organized as follows. Section 2 presents the IoT technology, and section 3 gives the solution of the addressed issues. Section 4 introduces the system analysis and design. Section 5 discusses experiments and the conclusion is given in section 6 .

\section{The loT technology}

Internet of Things technology is an important technological solution in this article. The IoT is a concept reflecting a connected set of anyone, anything, anytime, anyplace, any service, and any network [6]. The IoT enables physical objects to see, hear, think and perform jobs by having them "talk" together, to share information and coordinate decisions [7]. It is proposed on the basis of the concept of the Internet, which is mainly to extend the network to items and to allow information exchange between items and items. Communication, a network concept for intelligent control, positioning, and management of objects [8-13]. Through the IoT technology, it is possible to analyze and optimize products and life more finely and dynamically and realize the organic integration of human society and matter. Some of the most promising applications include improved management of global supply chain logistics, product counterfeit detection, manufacturing automation, smart homes and appliances, e-government (electronic official documents and currency), improved integrated vehicle health management, and e-health (patient monitoring and patient records) [14].

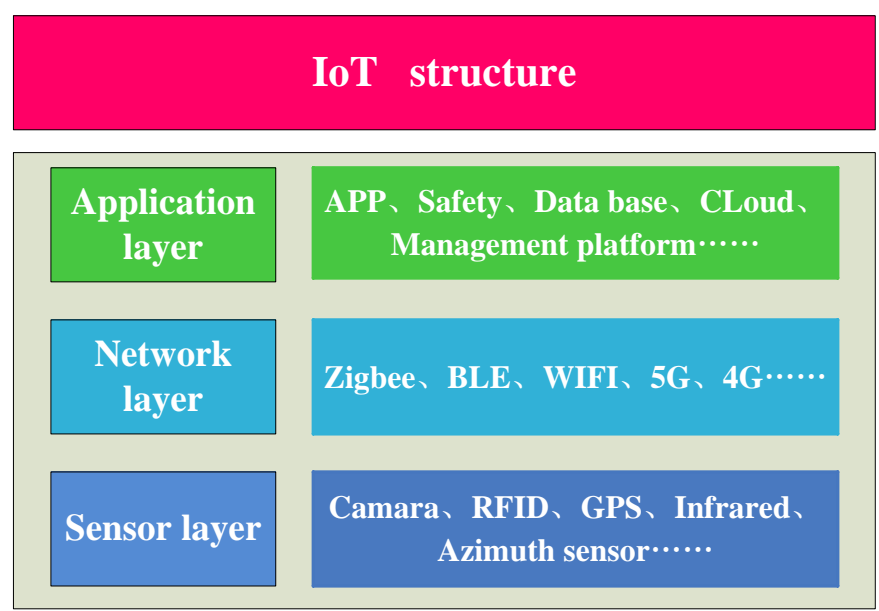

Fig. 1. The hierarchy of the Internet of things. 
As an extension of the Internet, the IoT is a network of connected objects. In the entire network, it integrates sensor technology, communication technology, computer network technology, and automatic control technology, etc., to achieve communication between things. The basic structure can be divided into three parts: the perception layer, the network layer, and the application layer, as shown in Fig. 1. The main task of the perception layer is to collect data. It is the basis of the IoT. It uses radiofrequency technology, smart sensors, and image acquisition technology to collect information about related objects at anytime, anywhere. The network layer mainly adopts wired and wireless technologies to form an ad-hoc network or transmit the underlying data to a network server. It must ensure realtime and accurate data transmission. The wired transmission is mainly through RS232, RS485, and optical fiber communication. Wireless transmission mainly includes Zigbee, BLE, WIFI, etc. It is also common to transmit the data collected by the perception layer to the network server through 3G / 4G / 5G technology. The application layer processes and analyzes the data to achieve the monitoring and control of the corresponding objects. The application layer includes databases, information processing, remote monitoring, and management of various platforms to achieve human-computer interaction and remote monitoring and control.

At present, the IoT technology in China has penetrated various industries of the national economy, such as transportation, security, logistics, energy, education, health, etc. And made the information management of these industries to enter a new stage. With the continuous improvement of China's economic development level, the pharmaceutical industry and medical service industry are showing a booming trend. There are more and more types of drugs, medical service coverage is increasing, and the medical security system is becoming more and more perfect. The medical and health, pharmaceutical, and other industries have put forward higher requirements.

Some researchers at domestic and abroad have also done some research in this area. Author Matthieu-P. Schapranow defines a formal approach for modeling data entities, events, and operations that correlate to the handling of pharmaceutical goods, and includes a quantitative analysis of the expected data amount and related costs for operating this service provider by applying our formal approach to a concrete pharmaceutical supply chain [15]. Author Chamekh $\mathrm{M}$ proposes a context-aware middleware for RFID based pharmacy supply chain, which aims to offer a deeper intelligence for the monitoring of the objects. A variant of Fosstrack middleware was proposed to offer a deeper intelligence in the RFID-based supply chain. RFID technology is becoming more and more important for tracing and tracking systems [16]. Author U. Barchetti describes the drug supply chain through AS-IS and TO-BE models in the article [17]. Current and possible future visions, build models for performing KPI analysis. The author Bevilacqua $\mathrm{M}$ analyzes the economic and qualitative evaluation of RFID technology in hospital drug management. The paper is mainly methodological, and does not deal with the technical feasibility of implementing RFID [18]. Author Dong-Ok Ko proposes an ultrahigh- frequency (UHF) band (center frequency $=915 \mathrm{MHz}$ ) RFID tag antenna for drug runout detection that can be attached to a drip chamber, The RFID tag antenna is designed to function as a sensor that alerts drug runout when a drug inside a drip chamber is completely consumed but does not work when a drug remains inside a drop chamber[19]. A series of studies have been conducted in the areas of supply chain management, RFID security management, model analysis, etc., and a series of analyses have been made in traditional drug management, hospital efficiency, etc., pointing out deficiencies, but providing a reference for technical operation. Less information. Once legal and social challenges and issues are resolved by consolidated legislation and improved RFID technology security standards, healthcare organizations are expected to speed up their pace to adopt RFID 
to patient related applications[20]. Author Chelliah, Ramachandran uses radio frequency identification (RFID) links and a wireless biomedical sensor network (WBSN) to monitor a set of intelligent pharmaceutical packages (iPackage) and a set of wearable biomedical sensor tags (iTag) [21]. In order to add value to RFID technology, various sensors are developed to be interfaced with RFID tags for monitoring different aspects of food quality [22].

In 2011, the author Zhuang Jianying proposed an RFID-based outpatient pharmacy warehousing and distribution system from a management perspective. The solution considers that the system's hardware construction consists of servers, electronic tag controllers, storage shelves, electronic tags, and handheld terminals. The electronic tags on the shelves record the order number, picking location, picking quantity, and completion status, so that the replenishment and inventory are realized through the information presented by the wireless handheld terminal. Pickers complete the picking task according to the color of the indicator light (representing a certain prescription number) and the quantity displayed on the electronic label [23]. Although this article only proposes an idea and does not solve the problem from a technical level, it provides a way for follow-up researchers. Author Peris-Lopez P and others propose an IS-RFID solution based on RFID technology. The demand is to equip nurses with a PDA incorporating an RFID reader. Other approaches require portable PCs to visit the inpatients or a wireless infrastructure [24]. Although this method can better achieve the safety of medication from the technical layer, the process is complicated, and lacks in drug management, hospital reconstruction cost, and convenience. In recent years, a context-aware RFID middleware distribution processes by combining event processing in the EPC global technology and semantic ontology is proposed [20]. The author proposes a framework for medical traceability systems from the perspective of middleware, which has certain practicality. However, it did not expand in depth to provide a specific implementation plan. In China, a better solution using RFID technology to locate and manage drugs is proposed. A roller ramp type medicine storage device is designed to cooperate with HIS to realize the positioning of medicines, and then automatically dispense medicine, which improved the efficiency of pharmacists in the medicine collection system and the degree of automation of medicine collection [25]. This solution using the IoT technology in the medicine taking system, improves the medicine dispensing method of the medicine dispensing equipment in the medicine dispensing system, improves the efficiency of medicine taking in the hospital, thereby improving the overall efficiency of the automated pharmacy, but lacks intelligent medicine. There is also a certain lack of traceability in the drug delivery inspection link.

\section{The system solution}

In order to solve the common problem of drug management and meet the needs of the current national and social development, this article proposes a drug distribution management plan based on the IoT technology, which makes up the insufficiency for the above several programs in drug management. First of all, flexible RFID tags are printed on the package box of drugs, and a series of information such as the name of the drug, dosage, raw materials, effects, production date, expiration date, and manufacturer can be stored in the label to avoid being tampered of the information so as to facilitate the identification, supervision, and investigation of fake and shoddy medicines. Secondly, in the hospital or drug store, the drug distribution management system, combined with the RFID reader can automatically identify the type, quantity, validity, authenticity, storage location, and other information of the drug, and realize intelligence in positioning and drug confirmation. It can significantly reduce the phenomenon of wrong delivery, missed delivery, fake medicines, expired medicines, etc., and reduce the 
medical accidents and drug complaints, and therefore improve management efficiency, reduce management costs, and control management risks.

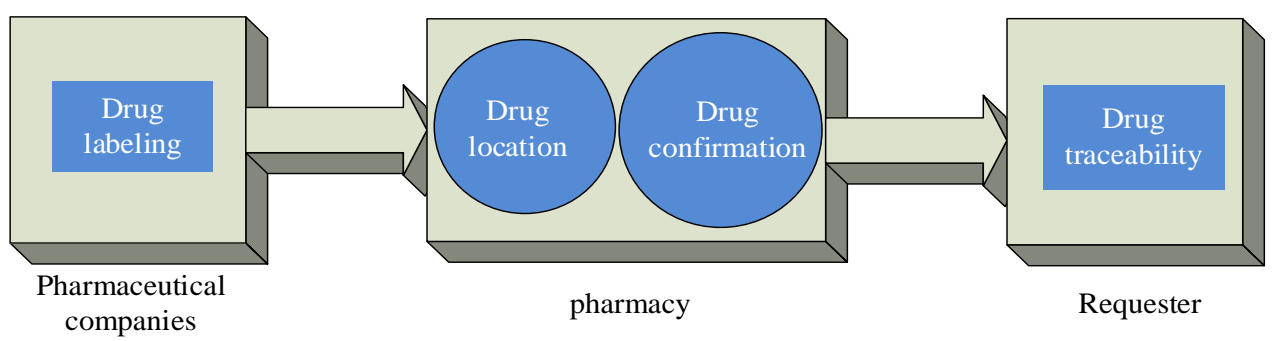

Fig. 2. Processing flowchart of the system.

The basic flow of this system is shown in Fig. 2. After the pharmaceutical manufacturer has finished the production of the drug, the flexible RFID tag is attached to the package. The label on each package is uniquely numbered. These labels are relatively small and flexible enough to accommodate various shapes of pharmaceutical packaging. When the electronic label can realize flexible folding and bending, it also means that the application range of the electronic label will be more widely expanded in many fields, including the medical field [26]. The label internally records the label number, drug name, and dosage, raw materials, effects, production date, expiration date, manufacturer, etc. When the medicine is purchased and entered the pharmacy, the medicine needs to deal with two tasks. One is the rational placement of the medicine and positioning, which is convenient for taking medicine. The second is to confirm the name, quantity, and quality according to the needs of customers or prescriptions when issuing medicines. After the customer or patient buys the medicine, if they find a problem, they can go to the pharmacy to check and trace the source through the medicine package. The hospital or pharmacy can also check and trace the source through historical records in its management system.

The RFID system of this scheme is divided into two interrelated subsystems: the RFID system of the medicine storage cabinet and the RFID system of the medicine distribution office. Drug storage cabinets are managed by the hospital's drug custodians, who plan and manage the types, quantities, storage environment, drug validity period, and entry and exit of drugs. As shown in Fig. 3, to facilitate the management, this scheme designs an RFID system and places the RFID reader on the medicine storage cabinet to supervise medicines. The data information read by the RFID reader is summarized to the protocol converter through the RS485 bus and then sent to the server for management. Storage cabinets in various domestic hospitals vary in size. Generally speaking, the height is 1 to 2.5 meters, the length is about 1 to 5 meters, and the width is 0.4 to 0.6 meters. The RFID reader is placed in the middle of the medicine storage cabinet, and LEDs are placed above the medicine storage cabinet. By appropriately setting of the RFID reading and writing area range, it is ensured that all medicines on the medicine storage cabinet (all with flexible RFID tags) can be detected. At the same time, it is not advisable to set the detection range too large, which will affect the results on the sideward medicine storage cabinet. During inventory counting, if the medicines in a certain area are tested by RFID, and the quantity or type does not reach the required inventory of the hospital's HIS system, the LED will emit red light to alarm. When there is a problem of expiration of drugs in a certain area, the LED in this area will light up yellow to remind. When the warehouse manager receives the instruction to fetch medicine from the HIS system, the LED in this area emits green light to remind the manager that there is a demand of taking medicine. The above various situations are reminded in the computer management software of 
the drug warehouse, and the data will be connected to the hospital's HIS system to realize the data upload and release.

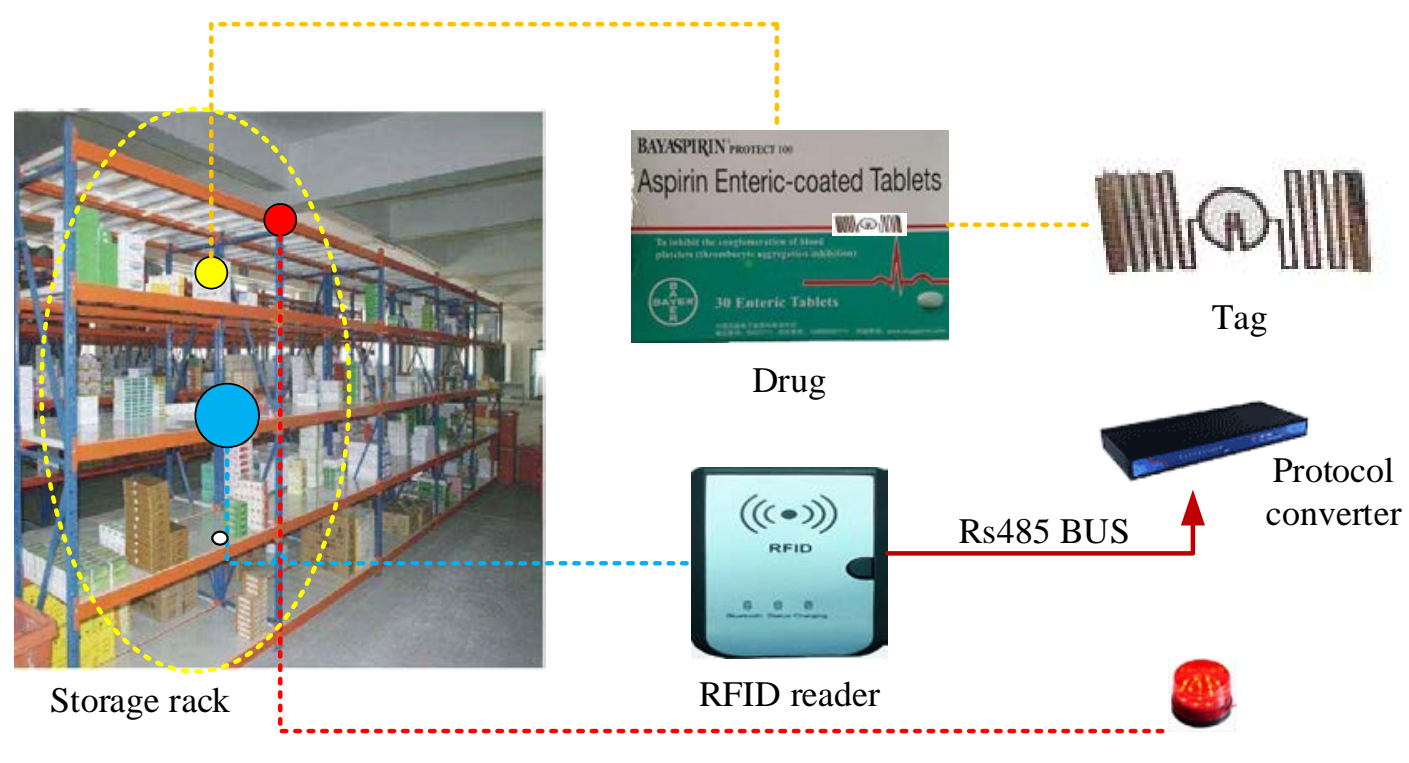

LED

Fig. 3. Medicine storage rack RFID system.

The RFID system of the drug distribution window is shown in Fig. 4. When medical workers receive the patient's medicine list sent by the HIS system, they will pick up the medicine from the medicine warehouse and pack the medicine in a plastic bag. After taking the medicine, the medicine needs to be checked before the distribution to the patients. In this scheme, a tray is designed for placing medicines, and an RFID reader is placed at the bottom of the tray. Put the plastic bag full of medicines on the pallet, and the RFID system will quickly read out the type, quantity, expiration date, and other information of all medicines. If the detected information is inconsistent with the medicine from the HIS system, an alarm message will be issued.

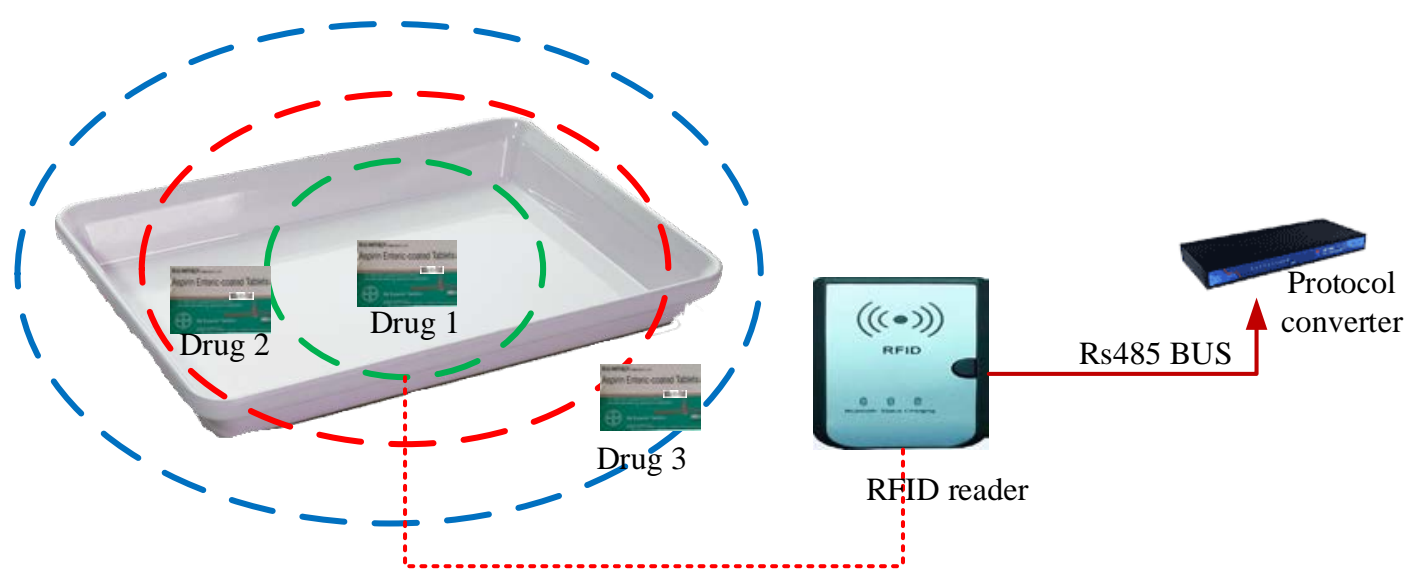

Fig. 4. Drug Distribution Office RFID system. 
In the process of verification of this solution, the most important issue that aroused our attention is the effective recognition range of the reader. When the effective recognition range is too small, missed picking will occur. For example, some drugs on the drug shelf in Figure 3 are not detected, causing statistical errors. When the effective recognition range is too large, repeated detection by two or more RFID readers will occur, causing data confusion. Similarly, as shown in Fig. 4, the range of the red circle is reasonable, the range of the blue circle is too large, and the range of the green circle is too small. Under normal circumstances, Drug 1 and Drug 2 should be identified. If the effective recognition range of the RFID reader is too small or too large, it will result in false detection of Drug 2 or excessive detection of Drug 3 of other patients. In order to be scientifically designed to ensure that the RFID system can correctly identify the drug information, the effective identification range of the reader must be controlled. After a series of tests, we have found a corresponding solution, which can effectively control the RFID recognition range through hardware and software, which will be discussed in subsequent articles.

\subsection{System loT Architecture}

The IoT technology is not a single technology, but a complete architecture that aggregates multiple technologies. According to the division of responsibilities, using the layered perspective to analyze the system of the IoT, and the system can be divided into three levels, the perception layer, the network layer, and the application layer. If the division from the information processing stage, it can be divided into three stages, information collection, information transmission, and information processing. These three stages don't correspond exactly to the three levels. Information collection is part of the function of the perception layer. Information transmission includes the short-distance transmission of the perception layer and the long-distance transmission of the network layer. Information processing is a function of the application layer. The architecture of this solution based on the IoT is shown in Fig. 5. The sensing layer is composed of electronic tags, RFID readers, etc., and is responsible for drug identification, positioning, and inspection functions. The network layer is implemented by Rs485 BUS, Protocol convert, and LAN. The application layer is composed of Server, Management Center, Pharmacy, and HIS systems, and realizes drug management, monitoring, and control.

Perception layer is the collector of target information, i.e. to obtain the status information of the target. In this system, RFID is used to realize the main functions of perception. The status information of a target is usually saved by a tag attached to the target. The status information on the tag can be obtained and written by the RFID reader. When the medicine is shipped from the factory, a series of information such as the name, dose, raw material, effect, production date, expiration date, and manufacturer of the medicine are stored in the tag through the reader, and this part of the data in the tag is write-protected to prevent from being tampered. Commonly used labeling technologies include barcodes, two-dimensional codes, two-dimensional or three-dimensional laser codes, RFID, and so on. Bar codes, two-dimensional codes, two-dimensional or three-dimensional laser codes are printed labels, which can be printed on the outer package. The biggest problem is that it is easy to be copied. RFID has quite good anti-counterfeiting characteristics. Each RFID tag has a unique number. The design and production of RFID tags can only be completed by IT hardware manufacturers with chip design capabilities and chip production lines. The design costs and manufacturing costs of hundreds of millions of yuan make the copycats timid. RFID tags contain specific circuit chips inside, so they are also called electronic tags. 


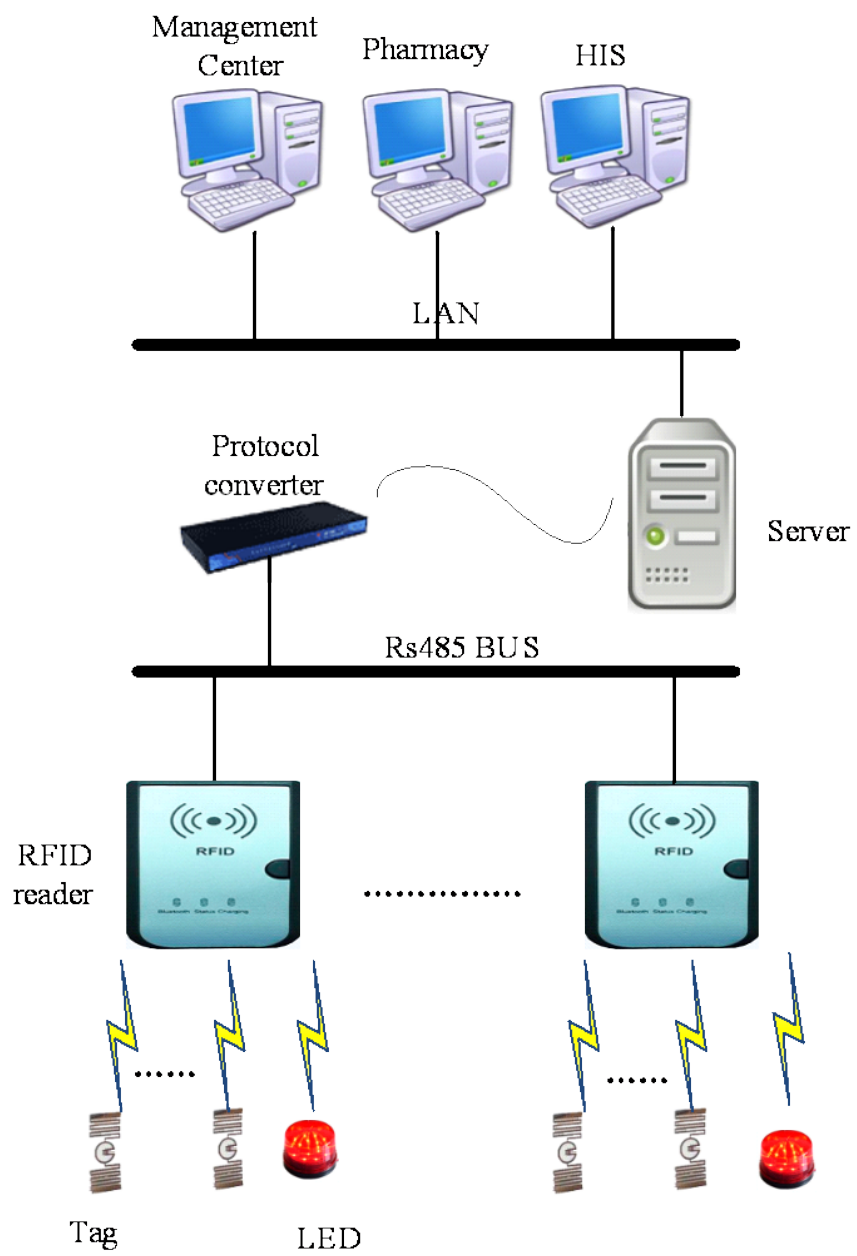

Fig. 5. Internet of things structure of the system.

The RFID readers are often placed in the medicine cabinets and drug sales windows of the pharmacy. These readers can collect the information in the label, and can also write some information, such as the code of the hospital or pharmacy that stores the drug, the time the drug was sold, and the operator. There are some differences in the functions of the RFID reader in the medicine cabinet and that of in the drug sales window. The former is focused on reading the basic information such as the name and quantity of the drug, and the focus is on determining the storage location of the drug. When the drug taker or the drug setting is set, the LED light at the corresponding location is illuminated to remind workers for easy searching.

Network layer is to collect the information of tags. The significance of a single collection of data is far less than that of a group, so they need to be passed together for storage, filtering, and analysis. In addition, for some collected data, it is necessary to judge its rationality and correctness by transmitting it to a remote server. The network layer provides the ability to transmit the collected status information over long distances. This transmission medium can be LAN, Zigbee, BLE, WIFI, 5G/4G/3G/2G, and other wired or wireless transmission methods. Rs485 BUS has mature applications in industrial control. It is suitable for multipoint transmission within a range that is not very far away. This is an important networking method for this solution after information collection. In order to facilitate the management and 
maintenance of the B / S architecture management software at the application layer, it is necessary to convert 485 to TCP / IP through protocol converter.

The main function of the application layer is to store, retrieve and process the collected information. When the amount of data is not large, ordinary databases can be used for storage and retrieval. In the face of massive information data, it is necessary to use distributed information storage technology and retrieval technology. Currently, popular cloud storage, cloud computing, and other technologies can complete complex tasks well. In addition to data storage and retrieval, another important function of the application layer is data processing, which is the specific application of data. Information such as medicines, locations, and pharmacies are read by various application systems, interacting with users through human-machine interfaces, and processing various business processes in various fields.

The three layers of perception layer, network layer, and application layer do not exist independently. They are related to each other and the information is shared among them. The transmitted data can be divided into two types: one type of status data, which is transmitted from the perception layer to the application layer through the network layer; the other type is control information, which is transmitted from the application layer to the perception layer through the network layer. For example, in the pharmacy management, the quantity information and location information are displayed by the LED indicator lights transmitted to the perception layer after the instructions are issued by the computer at the application layer.

\subsection{Advantages of RFID}

Currently, barcodes and QR codes are widely used, such as supermarket products, books, Alipay receipts and payments, WeChat receipts and payments, and identification of individuals and web addresses. Bar codes and QR codes are often used. They are low-cost and have a series of advantages. Why do we choose RFID at the sensing layer?

The bar code is a pattern composed of black and white stripes. The black stripes and white stripes represent binary 0 or 1 , respectively, and they are encoded to represent numbers, characters, and other information. The bar code is one-dimensional and only records horizontal information, not vertical information. The QR code is an upgraded version of the barcode, which records both horizontal and vertical information. The principle is the same. Scanners detect different reflected light from different emission areas through optical sensors for identification.

Barcode and two-dimensional code have a series of advantages such as fast input speed, high reliability, large information collection, and low cost, which are widely used. However, there are still some obvious shortcomings, and it is difficult to meet the current requirements for regulatory management and monitoring of drugs in the country. (1) Bar codes can only identify one type of product, but cannot identify single products, and cannot realize real-time tracking and tracing of the source of products. (2) Bar code is a visual transmission technology. Optical scanners must "see" the bar code to read it, which indicates that people usually have to align the bar code with the scanner to be effective. (3) The traditional bar code is an index code without classification and attribute information. It cannot implement applications such as classification queries and statistics. It must contact the database in real time to find complete description data from the database. The application is limited. It can be said that barcode technology can only be applied to the field of circulation (information management of business flow and logistics), it cannot transparently track and run through the supply chain process, realize traceability and traceability, and lack of supervision capabilities. 
With the advent of RFID technology, these defects can be better solved. The full name of RFID is Radio Frequency Identification, that is, radio frequency identification. RFID technology is often compared with bar codes and two-dimensional codes, and some even call RFID "smart bar codes." RFID's capabilities in automatic identification and data collection are far superior to barcodes and two-dimensional codes. It can not only store more information, is suitable for complex and harsh environments, can be invisible to humans, or even without a network. Work in the environment [24-31]. The chip-based on RFID technology has an embedded wireless transmission function and can store a certain amount of data. It uses the wireless radio frequency method and can perform non-contact two-way communication with the outside world for data storage and acquisition. The current manufacturing technology of RFID chips is relatively mature and has a high degree of integration. The volume can be made very small. It can be embedded in labels of various shapes, attached to target objects, and mark the status and attributes of target objects. Each RFID chip has a globally unique identification code, which is used to mark the uniqueness of the RFID chip. In addition, a self-destruct mechanism is provided to mimic illegal reuse. RFID technology is a wireless communication method where objects wearing RFID tags (microchips) are identified and tracked utilizing RF waves. The RFID system has two basic components: a memory microchip (tag) to store electronic information and an antenna-connected reader network to receive and analyze signals. A wireless sensor is used as well [32-41].

An application system built using RFID technology should include at least three parts: (1) RFID tags, (2) RFID sensors, and (3) application software (data acquisition middleware). When designing, many manufacturers integrate RFID sensors with application software and provide software interfaces for use. We call them RFID readers.

There are usually two types of RFID tags. One type of RFID tag can obtain the driving current through electromagnetic induction, but the amount of current obtained in this way is limited, so it also limits the distance that the RFID tag sends information and the amount of information data. To compensate for this, some RFID tags can be individually configured with a power supply to increase the distance and amount of information sent, and can also actively send information to the sensor without waiting for the sensor's induction signal, Called active tags.

Later, in order to combine the advantages of passive RFID tags and active RFID tags, a combined form of RFID tags appeared, which is also equipped with a battery, but uses less electricity than an active RFID tag. The battery power is only used to reply information to the sensor and is not responsible for receiving the detection signal from the sensor. When receiving, the electromagnetic induction method of passive RFID tags is still used. The advantage of this is that it can have better response speed, and can reduce the size and cost of the battery under the same service life.

Table 1. Operational frequencies in RFID

\begin{tabular}{ll}
\hline \multicolumn{1}{c}{ Operational Frequency } & \multicolumn{1}{c}{ Band } \\
\hline Low Frequency & $125 \mathrm{KHz}$ and $134.2 \mathrm{KHz}$ \\
High Frequency & $13.56 \mathrm{MHz}$ \\
Ultra-High Frequency & $433 \mathrm{MHz}, 860 \mathrm{MHz} \sim 960 \mathrm{MHz}$ \\
Microwave & $2.45 \mathrm{GHz}, 5.8 \mathrm{GHz}$ \\
\hline
\end{tabular}

Depending on the use environment, the operating frequency of RFID tags is different, and the characteristics displayed are also different. There are currently four main types of operating frequencies, low frequency, high frequency, ultra-high frequency, and microwave, 
as shown in Table 1.

Compared with barcode, two-dimensional code, and other technologies, RFID has a series of advantages such as high read-write efficiency, strong anti-counterfeiting ability, easy source tracing, and information control. This system uses RFID as an important part of the pharmacy management solution. That is, the two important modules responsible for drug positioning and drug confirmation in the pharmacy are organized into an EPC system. EPC system is a complex and comprehensive system, including RFID, EPC coding, network, communication protocols, etc. RFID is an important part of it. EPC is one of the application fields of RFID technology. Only specific low-cost RFID tags are suitable for EPC systems [42-44]. Through the introduction of EPC/RFID technology into production management, automatic and real-time entry of product information can be achieved, and the causal information of all processes and costs incurred for each product can be accurately recorded.

It can be seen that RFID technology has a series of advantages such as high anti-counterfeiting, information control, and tracking and tracing. In the process of using, it can effectively reduce the cost of drugs and effectively control the production of counterfeit and inferior drugs. The RFID tag contains an electronic product code (EPC) that gives each drug a unique identification code, which is critical in the application of drug management. A series of serial numbers represented by traditional barcodes or two-dimensional codes must be connected to the back-end database to obtain asset information, while RFID tags have the function of storing data. Users can encode the tags according to their own needs, even if there is no network connection. You can also access drug information in cases. In addition, the reading speed of RFID is much faster than two-dimensional codes and barcodes, which can reach 100 times. The application of bar codes does have obvious limitations-you can only scan it when you see the bar code. This means that the asset should be placed with the side facing the bar code facing outwards for easy manual scanning. RFID doesn't have to be so troublesome. You don't need to see the tag when reading the tag. As long as it is within the read and write distance specified by the tag, you can easily scan all tags with a handheld or fixed reader, and get information quickly and accurately. Therefore, we used RFID technology in the design process, selected the JRM2030 core chip, which has an operating frequency of $840 \sim 960 \mathrm{MHz}$, and developed and designed using the EPC C1 GEN2/ISO 18000-6C protocol.

\section{System analysis and design}

In order to test the rationality of the system design plan and show its practical application value, it is necessary to analyze the functional requirements of the system. Then we need to design and develop specific hardware circuits and software programs, which consists of conducting a series of tests, finding out the main problems, and further improving program measures. Of course, there are some conditions in the plan, such as the self-labeling of medicines transported out of the factory, which is currently unsatisfactory in society. With the further development of the information society, it can be met in the future. In the experiment, we have to adopt a flexible solution, by manually adding tag information to simulate to meet its basic functions.

\subsection{Functional requirements analysis}

In the drug management system, there are mainly three types of user roles, drug inventory administrator, drug issuer, and system administrator. 
The main function of the drug inventory manager is to store, inventory, and destroy drugs. This system is only designed for the management after drug purchase and does not involve the production stage and logistics stage. We expect the best condition is that the drugs have only RFID tags when the pharmaceutical companies leave the factory, but in the current situation, almost no RFID tags are attached when the drugs are produced. Therefore, during the experiment, after purchasing, we need to attach RFID tags to the smallest selling unit of the medicines in storage. The coding in the RFID tag adopts the EPC 128-bit type III standard to encode the smallest sales unit of the drug, and the RFID tag is written as the unique code of the drug. The attribute information and status information of the medicine is stored in the medical data server. When the medicine is put into the warehouse, the attribute information and status information of the medicine need to be entered first, then the RFID tag is pasted and the code is written.

One month before the expiration of the medicine, a reminder is started to guarantee the quality of medicines. When the RFID system in the pharmacy detects that certain medicines will expire in a month, the LED emits yellow light. For medicines within five days of validity, they are regarded as expired medicines, and the red light is on to remind the drug inventory manager to prompt the handling. At the same time, after the RFID system in the pharmacy scanning the drugs in all directions, it can identify the classified inventory information of the drugs, and inform the drug inventory administrator of the inventory quantity and quality, and remind the drug inventory administrator to purchase and destroy the drugs operating.

Drug distributors need to hand out medicines according to the doctor's prescription. The main functions include printing the medicine picking list and medicine out of the warehouse. The medicine picking list is printed to reduce the medicine picking time of the medicine issuer. According to the medicine location information stored in the database and the medicine list on the doctor's prescription, the medicine issuer is instructed to quickly obtain the medicines needed. The function of medicines out of the warehouse is to record the information of the medicines out of the warehouse. Before distributing the medicines, the medicine dispensers place the medicines of each person on the RFID system of the medicine distributing place for inspection. The system also needs to check the quality and other information, and judge whether it is consistent with the drug picking list and issue relevant prompt information.

The main function of the system administrator is the management and maintenance of the system, mainly including system user management and data management. User management is to maintain users who can be used in the system, including adding users, deleting users, and resetting user passwords. Data management is to ensure the security of the system data.

\subsection{System design}

The core part of the design of the hardware system is the design of the RFID reader. This system uses the advanced RFID special RF chip JRM2030, combined with digital signal processing technology for design. It has an accurate RSSI value for easy positioning analysis. Because RFID in this system has two different application scenarios of drug positioning and drug confirmation, the two places in the design should be processed differently according to the actual situation. Considering the design cost, development cycle, and easy maintenance, both applications use the same design. Through the adjustment of the power level, two different applications are satisfied. The positioning of the drugs by the reader in the pharmacy is generally designed to be realized in the range of 0.5-4 square meters. Too large distance is conducive to neither classification of drugs nor location of the drugs. The identification range of the device is generally set between 0.1-0.5 square meters. If the range is too large, the drugs of the other shelves may be misrecognized for processing. Therefore, the range of the 
identification of the RFID reader can be adjusted by the transmitting power, therefore, the above two requirements can be met at the same time.

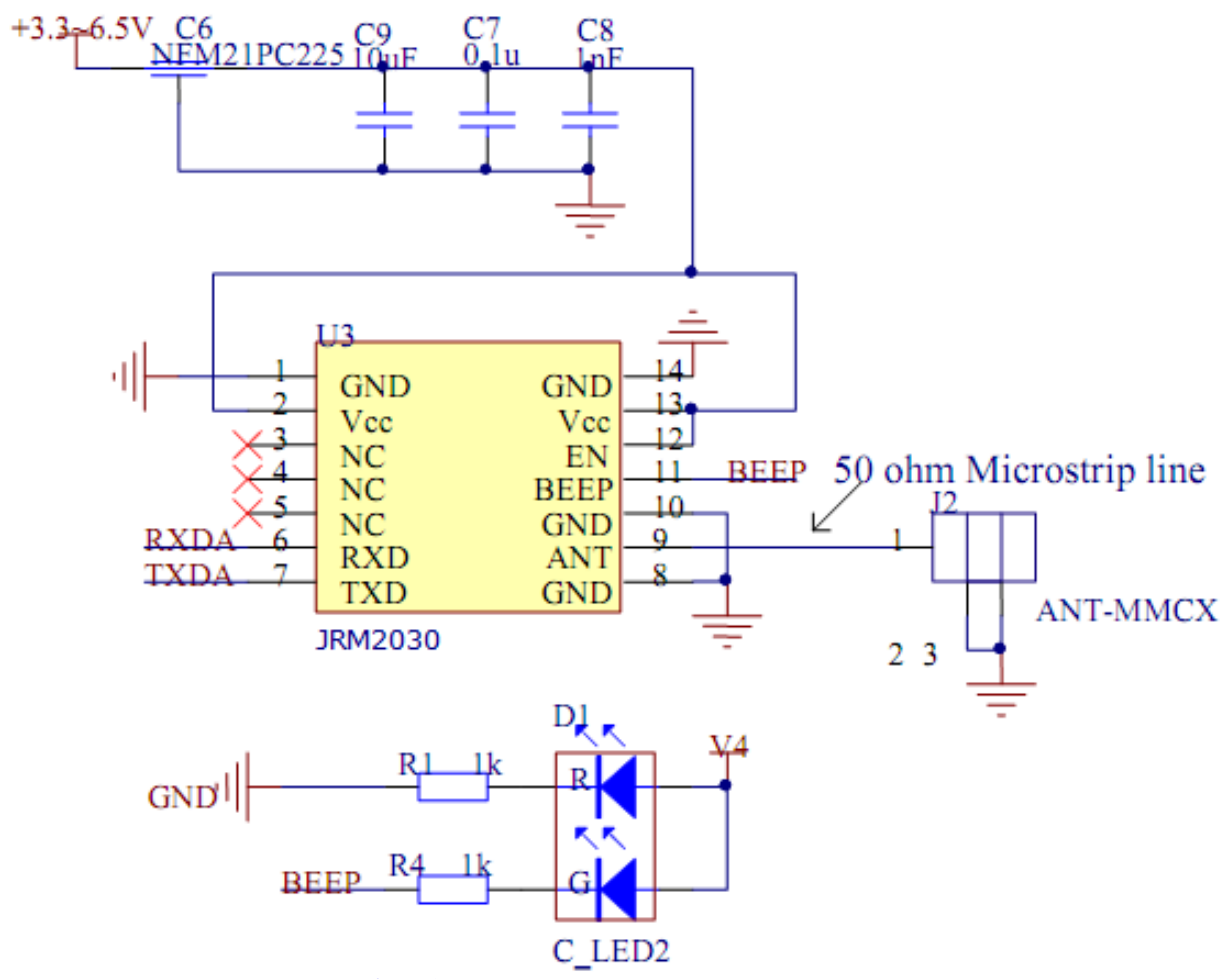

Fig. 6. Core module circuit diagram.

The core circuit part of the reader is shown in Fig. 6. For long-distance communication through RS485, it is necessary to connect pins 6 and 7 to MAX485. It is recommended to use a tantalum capacitor of $100 \sim 470 \mathrm{uF}$ for the VCC port to reduce the power amplifier during transmission. For the traction of the power supply, the antenna should use 50-ohm micro strip line.

In terms of the requirements of software design, the entire software is divided into 3 information maintenance modules, which are related to each other. (1) Maintenance of basic drug information. This information includes drug ID, drug name, approval number, main raw materials, main ingredients, functions, usage, storage method, specifications, shelf life, adverse reactions, precautions, manufacturer, production batch number, production date, production address, etc. (2) Maintenance of drug management information. This information includes drug purchase price, batch quantity, sale price, agent, contract number, time of storage, time of storage, number of drug collection order, attending doctor, drug distributor, etc. (3) Maintenance of personnel management information. This information includes: drug inventory managers, drug distributors, attending doctors, system administrators, etc.

Table 2. Operational frequencies in RFID

\begin{tabular}{clc}
\hline No & \multicolumn{1}{c}{ Function } & Result \\
\hline 1 & Software installation, uninstallation & Full \\
2 & Software interface display & Normal \\
3 & Information maintenance & Normal \\
4 & Management authority & Normal \\
\hline
\end{tabular}




\begin{tabular}{lll}
\hline 5 & Database & Normal \\
6 & Tag identification & Normal \\
7 & HIS ACCESS & Normal \\
8 & Alarm & Normal \\
\hline
\end{tabular}

Finally, the software system is realized under C++ and QT development environments. The functional tests are carried out and the test results are shown in Table 2, each function of the software meets the design requirements.

Some basic conditions during our experiment are shown in Fig. 7. Through hardware design and software development, a system test was completed, the expected results were achieved, and the conclusion was verified.

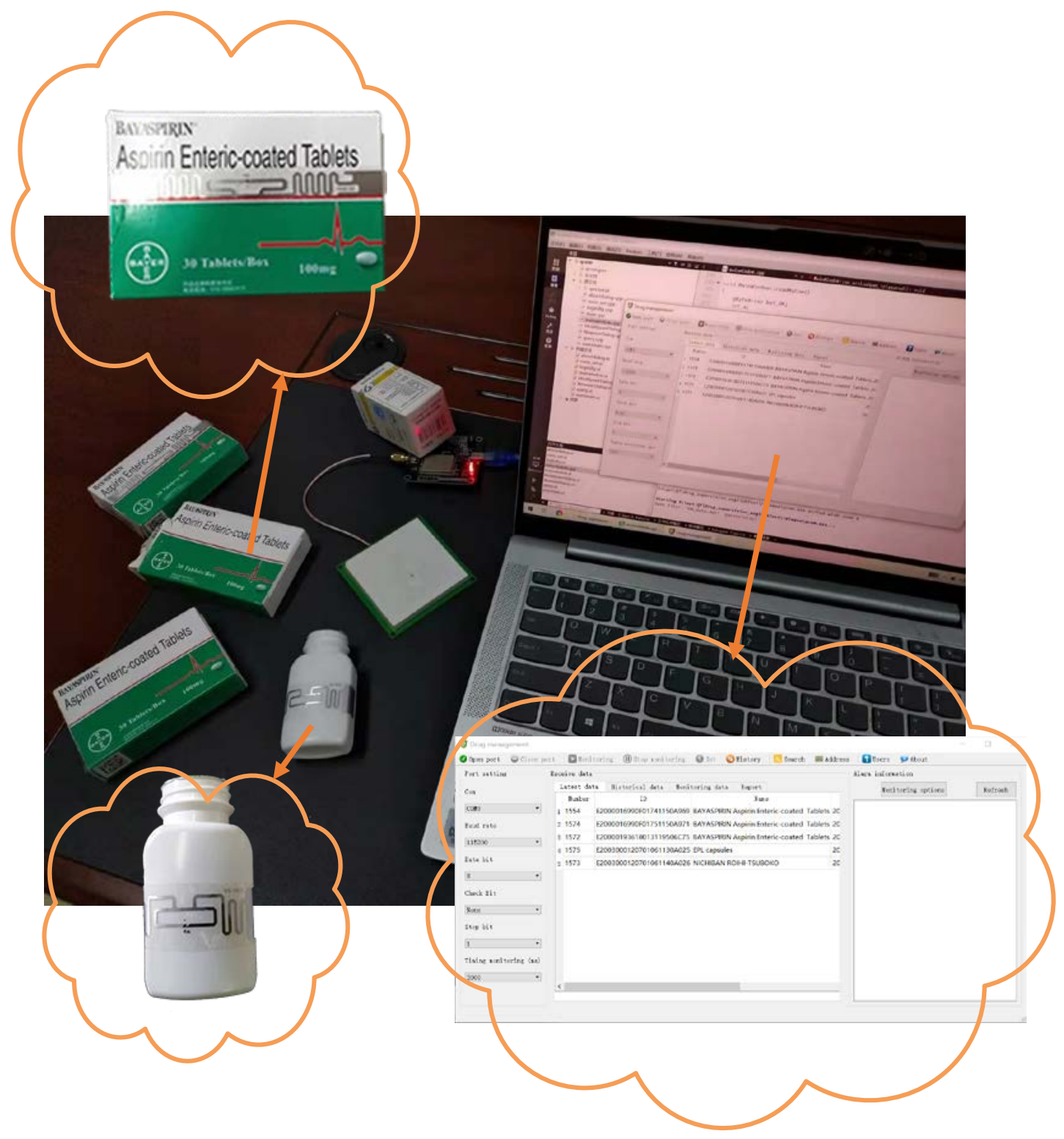

Fig. 7. The experiment. 


\section{Experimental tests and discussion}

To ensure better results, we conducted a series of experiments to optimize the design of the system. The identification distance of the RFID readers is an important parameter. In the case of no load, the test is performed with a default power consumption of 20dB. The effect is shown in Fig. 8. Within 3 meters, the labels can basically be identified without omission. Attenuation occurs between 3-5 meters, and the labels cannot be identified within the specified time range, and there is basically no use value beyond 5 meters.

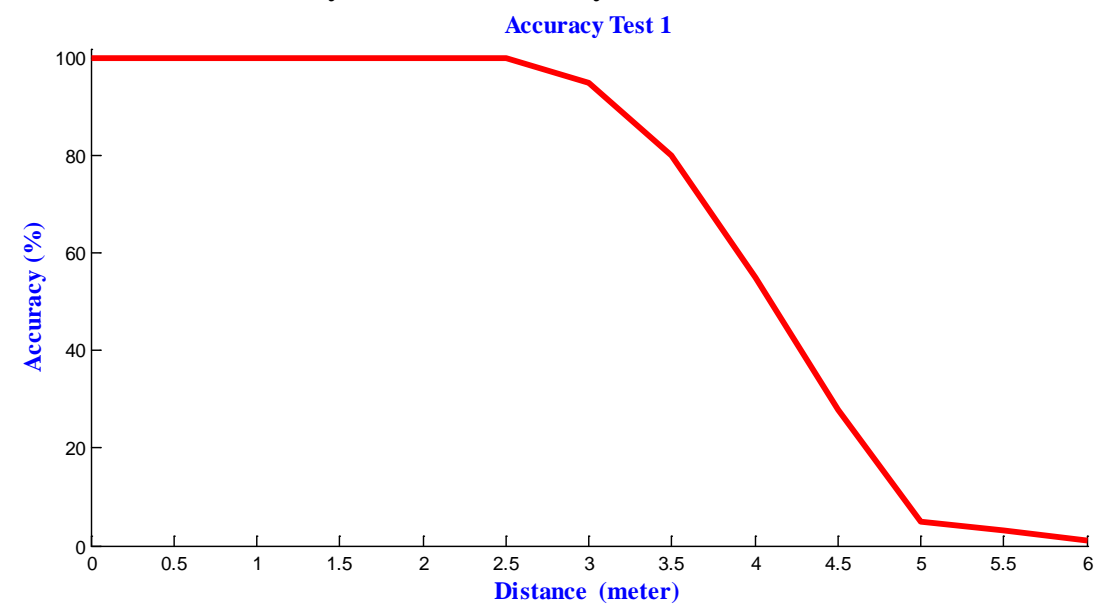

Fig. 8. Analysis of relationship between accuracy and distance.

The relative angle between the reader antenna and the tag antenna has a certain effect on the reading distance of the tag. After multiple tests of relative offset angle adjustment, the fitting curve was obtained as shown in Fig. 9. The tests show that within 30 degrees, the label reading effect is very good. Between 30 degrees and 60 degrees, the reading rate drops, but it is acceptable. Above 60 degrees, the tag reading rate will drop significantly, with a greater impact. In the actual application, the reader should adjust the power according to the recognizable effective range to ensure the reliable reading and writing of the label. It is recommended that the angle is set within 30 degrees. In addition, the effect of bending of the flexible label and sticking it on the bottle-shaped medicine package is worse than that of the flat label on the plate-shaped medicine package. In practice, these actual factors should also be considered for debugging.

Accuracy Test 2

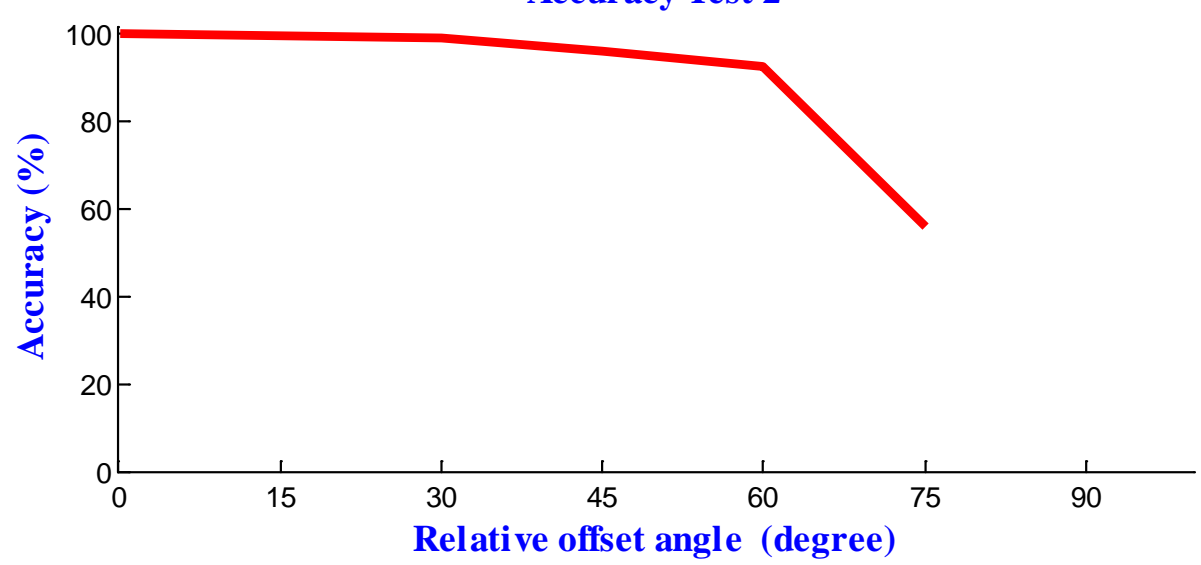

Fig. 9. Analysis of relationship between accuracy and relative offset angle. 
In addition to accuracy, we also conducted a simple test of the reading and writing rate of the RFID reader. Generally speaking, when medicines are issued, each patient or customer receives between 1-30 pieces of medicines at one time, so we have selected 5 and 30 labels for 8 repetitive read and write tests, the result is shown in Fig. 10. Experiments show that the reading and writing rates of 5 tags and 30 tags are basically the same, and each tag takes about $3 \mathrm{~ms}$. Experiments also show that the faster read and write speed can meet the application requirements of the system, and has a greater advantage than the solution using the two-dimensional code.

When RFID is used to locate medicines, more power is needed to save costs and reduce the number of readers used. When RFID is used to confirm medicines, more attention is paid to accuracy and anti-interference. The power of the reader should be set a little lower to reduce the false identification of nearby labels. In addition, when multiple tags are read at the same time, the anti-collision algorithm of multiple tags should be refined to improve efficiency and accuracy.

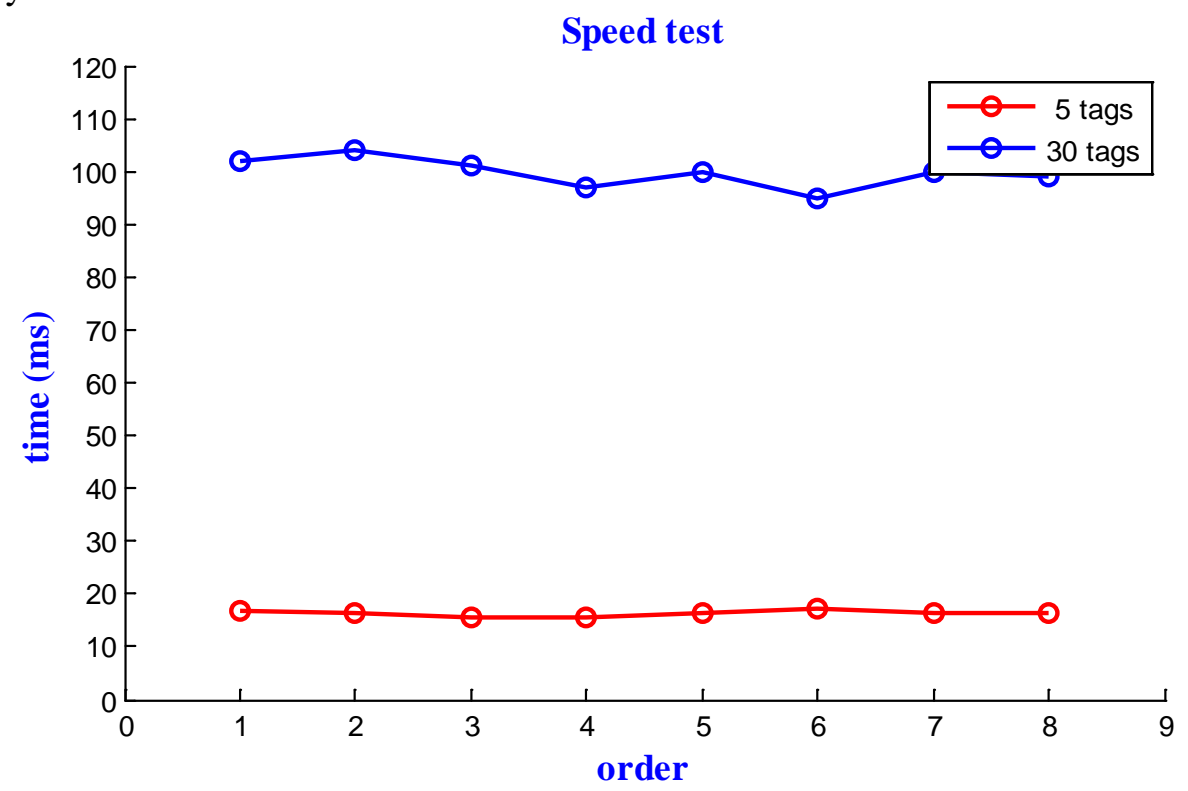

Fig. 10. Speed test

\section{Conclusion}

This system changes the original hospital pharmacy working mode, realizes the automatic identification of drugs through the IoT technology and makes the order of drug storage and distribution, reduces the error of artificial drug distribution. The proposed system improves the efficiency of drug distribution in pharmacies, saves the cost of human resources in the hospital, and can realize drugs tracking and tracing to reduce the occurrence of medical accidents and save unnecessary losses. This system also realizes the functions of automatic drug identification, intelligent medicine retrieval system, monitoring management, and alarm system. It involves technical aspects such as hardware circuit design, software system development, database system design, and communication networking. Through the use of RFID technology, drug identification and positioning are realized, which improves the efficiency of pharmacists in taking medicines and improves the degree of automation of taking 
medicines; monitors the quality and quantity of medicines through monitoring and alarm systems to ensure the accuracy of medicine operations quality, rationality, standardization and reliability; the optimization of the medicine dispensing system software improves the stability, accuracy and efficiency of the medicine dispensing system, ensures that the equipment can accurately receive prescription information, and avoids medicine dispensing errors; The wireless and wired communication technology realizes the networking of the hospital's HIS system and the drug management system, greatly reducing the round trip of patients or their families, improving the relationship between doctors and patients, and improving the efficiency of multiple parties.

The remarkable advantage of the system is that it can achieve traceability, anti-tampering information, improve the defects of the aforementioned two-dimensional codes and other solutions, and meet the needs of social development. Meanwhile, through technical improvements and updates, the low-cost, easy way to connect to the current hospital HIS system can be achieved. The system improves the efficiency of doctors and reduces the pressure on doctors. It can also greatly reduce the accidents of wrong prescriptions, provide patients with efficient and safe medication services, and reduce the cost of human resources in hospitals, bringing huge social benefits and economics benefit. At the same time, the program also has some problems that need to be further studied and solved in the later stage: First, security issues should be focused on, including the leakage of personal information, data security of the IoT system and network security. The second is that the system involves multiple levels such as drug manufacturers, agents, hospitals, and individuals, and each level has many individuals. In particular, drug manufacturers are affected by various factors such as geography, corporate culture, laws and regulations, local policies, and corporate interests. How to achieve multi-party coordination and unification of EPC coding standards and provide convenient services to society also requires national and social considerations. The third is that the technical level of the system has certain restrictions on obstacles and planning and layout. Looking for better and more optimized solutions to reduce interference factors also needs to be considered later.

\section{References}

[1] https://www.nmpa.gov.cn/directory/web/nmpa/zwgk/tjxx/tjnb/20170523155301990.html

[2] https://www.nmpa.gov.cn/directory/web/nmpa/zwgk/tjxx/tjnb/20180402152301596.html

[3] https://www.nmpa.gov.cn/zwgk/tjxx/tjnb/20190509120001890.html

[4] https://www.nmpa.gov.cn/yaopin/ypggtg/ypqtgg/20190827171501407.html

[5] S. M. Riazul Islam, D. Kwak, M. Humaun Kabir, M. Hossain, K. Kwak, "The Internet of Things for Health Care: A Comprehensive Survey," IEEE Access, vol. 3, pp. 678-708, 2015. Article (CrossRef Link)

[6] A. Al-Fuqaha, M. Guizani, M. Mohammadi, M. Aledhari, M. Ayyash, "Internet of Things: A Survey on Enabling Technologies, Protocols, and Applications," IEEE Communications Surveys \& Tutorials, vol. 17, no. 4, pp. 2347-2376, 2015. Article (CrossRef Link)

[7] Leng jing, "Design and implementation of Internet of things gateway platform based on M2M," Xi'an University of Electronic Science and technology, 2014.

[8] D. Bandyopadhyay and J. Sen, "Internet of Things: Applications and Challenges in Technology and Standardization," WIRELESS PERSONAL COMMUNICATIONS, vol. 58, no. 1, pp. 49-69, 2011. Article (CrossRef Link)

[9] W. D. D. V. Alessio Botta, "Integration of Cloud Computing and Internet of Things: a Survey," Future Generation Computer Systems, vol. 56, pp. 684-700, 2016. Article (CrossRef Link) 
[10] A. Gluhak, S. Krco, M. Nati, D. Pfisterer, N. Mitton, T. Razafindralambo, "A survey on facilities for experimental internet of things research," IEEE COMMUNICATIONS MAGAZINE, vol. 49, no. 11, pp. 58-67, 2011. Article (CrossRef Link)

[11] J. Lin, W. Yu, N. Zhang, X. Yang, H. Zhang, W. Zhao, "A Survey on Internet of Things: Architecture, Enabling Technologies, Security and Privacy, and Applications," IEEE Internet of Things Journal, vol. 4, no. 5, pp. 1125-1142, 2017. Article (CrossRef Link)

[12] A. Zanella, N. Bui, A. Castellani, L. Vangelista, M. Zorzi, "Internet of Things for Smart Cities," IEEE Internet of Things Journal, vol. 1, no. 1, pp. 22-32, 2014. Article (CrossRef Link)

[13] C. Perera, A. Zaslavsky, P. Christen, D. Georgakopoulos, "Context Aware Computing for The Internet of Things: A Survey," IEEE Communications Surveys \& Tutorials, vol. 16, no. 1, pp. 414-454, 2014. Article (CrossRef Link)

[14] T. Sánchez López, D. C. Ranasinghe, M. Harrison, D. McFarlane, "Adding sense to the Internet of Things," Personal and Ubiquitous Computing, vol. 16, no. 3, pp. 291-308, 2012. Article (CrossRef Link)

[15] M. Schapranow, A. Zeier, H. Plattner, "A Formal Model for Enabling RFID in Pharmaceutical Supply Chains," in Proc. of 2011 44th Hawaii International Conference on System Sciences, pp. 1-10, 2011. Article (CrossRef Link)

[16] M. Chamekh, S. E. Asmi, M. Hamdi, T. Kim, "Context Aware Middleware for RFID Based Pharmaceutical Supply Chain," in Proc. of 2017 13th International Wireless Communications and Mobile Computing Conference (IWCMC), pp. 1915-1920, 2017. Article (CrossRef Link)

[17] U. Barchetti, A. Bucciero, M. De Blasi, A. L. Guido, L. Mainetti, L. Patrono, "Impact of RFID, EPC and B2B on traceability management of the pharmaceutical supply chain," in Proc. of 5th International Conference on Computer Sciences and Convergence Information Technology, pp. 58-63, 2010. Article (CrossRef Link)

[18] M. Bevilacqua, F. E. Ciarapica, G. Mazzuto, C. Paciarotti, "The impact of RFID technology in hospital drug management: An economic and qualitative assessment," International Journal of RF Technologies, vol. 4, no. 3-4, pp. 181-208, 2013. Article (CrossRef Link)

[19] Dong-Ok Ko, Jong-Myung Woo, "Design of a Small Radio Frequency Identification Tag Antenna Using a Corrugated Meander Line Applicable to a Drug Runout Sensor System," Journal of Electromagnetic Engineering and Science, vol. 18, pp. 7-12, 2018. Article (CrossRef Link)

[20] Haddara, M and A. Staaby, "RFID Applications and Adoptions in Healthcare: A Review on Patient Safety," Procedia Computer Science, vol. 138, pp. 80-88, 2018. Article (CrossRef Link)

[21] Chelliah, Ramachandran, et al., "Development of Nanosensors Based Intelligent Packaging Systems: Food Quality and Medicine," Nanomaterials, vol. 11.6, p. 1515, 2021. Article (CrossRef Link)

[22] DOBRUCKA, RENATA, PRZEKOP, ROBERT, "New perspectives in active and intelligent food packaging," Journal of Food Processing and Preservation, vol. 43, pp.1-9, 2019. Article (CrossRef Link).

[23] Zhuang jianying, "Design and implementation of outpatient pharmacy warehouse distribution system based on RFID," Logistics and procurement in China, 2011(18), 60-61, 2011.

[24] P. Peris-Lopez, A. Orfila, A. Mitrokotsa, J. C. A. van der Lubbe, "A comprehensive RFID solution to enhance inpatient medication safety," INTERNATIONAL JOURNAL OF MEDICAL INFORMATICS, vol. 80, no. 1, pp. 13-24, 2011. Article (CrossRef Link)

[25] Yang jiangbing, "Research on rapid drug delivery system of modern pharmacy based on Internet of things technology,” Beijing: Beijing University of Posts and telecommunications, 2015.

[26] Z. Liu, M. Wang, S. Qi, C. Yang, "Study on the Anti-Theft Technology of Museum Cultural Relics Based on Internet of Things," IEEE Access, vol. 7, pp. 111387-111395, 2019. Article (CrossRef Link)

[27] Z. Zhao, "A Secure RFID Authentication Protocol for Healthcare Environments Using Elliptic Curve Cryptosystem," JOURNAL OF MEDICAL SYSTEMS, vol. 38, no. 5, 2014. Article (CrossRef Link) 
[28] S. Amendola, R. Lodato, S. Manzari, C. Occhiuzzi, G. Marrocco, "RFID Technology for IoT-Based Personal Healthcare in Smart Spaces," IEEE Internet of Things Journal, vol. 1, no. 2, pp. 144-152, 2014. Article (CrossRef Link)

[29] B. P. Rosenbaum, "Radio Frequency Identification (RFID) in Health Care: Privacy and Security Concerns Limiting Adoption," JOURNAL OF MEDICAL SYSTEMS, vol. 38, no. 3, 2014. Article (CrossRef Link)

[30] J. Wang, C. Floerkemeier and S. E. Sarma, "Session-based security enhancement of RFID systems for emerging open-loop applications," Personal and Ubiquitous Computing, vol. 18, no. 8, pp. 1881-1891, 2014. Article (CrossRef Link)

[31] G. P. Joshi, S. Acharya, C. Kim, B. Kim, S. W. Kim, "Smart Solutions in Elderly Care Facilities with RFID System and Its Integration with Wireless Sensor Networks," International Journal of Distributed Sensor Networks, vol. 10, no. 8, 2014, Art. no 713946. Article (CrossRef Link)

[32] C. Li, C. Weng and C. Lee, "A Secure RFID Tag Authentication Protocol with Privacy Preserving in Telecare Medicine Information System," JOURNAL OF MEDICAL SYSTEMS, vol. 39, no. 8, 2015. Article (CrossRef Link)

[33] A. Coustasse, P. Meadows, R. S. Hall, T. Hibner, S. Deslich, "Utilizing Radiofrequency Identification Technology to Improve Safety and Management of Blood Bank Supply Chains," Telemedicine and e-Health, vol. 21, no. 11, pp. 938-945, 2015. Article (CrossRef Link)

[34] C. Jin, C. Xu, X. Zhang, F. Li, "A Secure ECC-based RFID Mutual Authentication Protocol to Enhance Patient Medication Safety," JOURNAL OF MEDICAL SYSTEMS, vol. 40, no. 1, 2016. Article (CrossRef Link)

[35] M. Gaynor and J. Waterman, "Design framework for sensors and RFID tags with healthcare applications," Health Policy and Technology, vol. 5, no. 4, pp. 357-369, 2016. Article (CrossRef Link)

[36] M. Benssalah, M. Djeddou and K. Drouiche, "Dual cooperative RFID-telecare medicine information system authentication protocol for healthcare environments," Security and Communication Networks, vol. 9, no. 18, pp. 4924-4948, 2016. Article (CrossRef Link)

[37] M. Martínez Pérez, G. Vázquez González and C. Dafonte, "Evaluation of a Tracking System for Patients and Mixed Intravenous Medication Based on RFID Technology," SENSORS, vol. 16, no. 12, pp. 2031, 2016. Article (CrossRef Link)

[38] V. J. Silva, C. E. M. Gomes, S. S. Santana, V. F. De Lucena, "Intelligent System for Medication Management in Residential Environments," IFAC-PapersOnLine, vol. 49, no. 30, pp. 171-174, 2016. Article (CrossRef Link)

[39] S. V. Zanjal and G. R. Talmale, "Medicine Reminder and Monitoring System for Secure Health Using IOT," Procedia Computer Science, vol. 78, pp. 471-476, 2016. Article (CrossRef Link)

[40] J. Akmal, M. Salmi, A. Mäkitie, R. Björkstrand, J. Partanen, "Implementation of Industrial Additive Manufacturing: Intelligent Implants and Drug Delivery Systems," Journal of Functional Biomaterials, vol. 9, no. 3, pp. 41, 2018. Article (CrossRef Link)

[41] M. Caldara, B. Nodari, V. Re, B. Bonandrini, "Miniaturized Blood Pressure Telemetry System with RFID Interface," Electronics, vol. 5, no. 3, pp. 51, 2016. Article (CrossRef Link)

[42] M. Martínez Pérez, G. Vázquez González and C. Dafonte, "Safety and Traceability in Patient Healthcare through the Integration of RFID Technology for Intravenous Mixtures in the Prescription-Validation-Elaboration-Dispensation-Administration Circuit to Day Hospital Patients," SENSORS, vol. 16, no. 8, pp. 1188, 2016. Article (CrossRef Link)

[43] R. Singh, E. Singh and H. S. Nalwa, "Inkjet printed nanomaterial based flexible radio frequency identification (RFID) tag sensors for the internet of nano things," RSC Advances, vol. 7, no. 77, pp. 44863-48597, 2017. Article (CrossRef Link)

[44] S. Utku, M. H. Özcanhan and M. S. Unluturk, "Automated personnel-assets-consumables-drug tracking in ambulance services for more effective and efficient medical emergency interventions," COMPUTER METHODS AND PROGRAMS IN BIOMEDICINE, vol. 127, pp. 216-231, 2016. Article (CrossRef Link) 

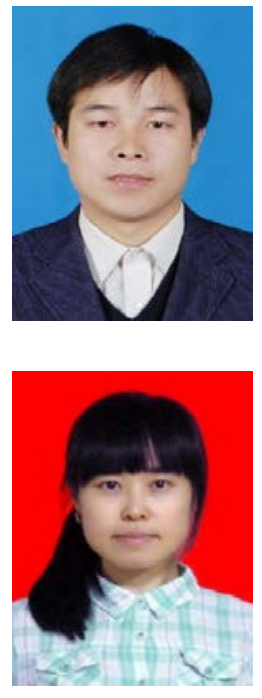

Zeliang Liu was born in 1980.He received the BS degree from Central China Normal University, Wuhan, China, in 2003 and the MS degree from Wuhan University Of Technology, Wuhan, China, in 2009. In 2019, he was a Visiting Scholar at the Wuhan University. He is currently an associate Professor in the School of Electronic and Information Engineering, Jiujiang University, China. His research interests include wireless sensor networks, Internet of Things, and information resources management.

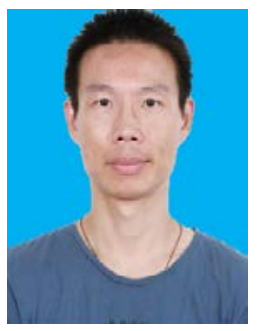

Hui Peng was born in 1973. He received the PhD from university of lyon1, France in 2011. He is currently a lecturer in the School of Electronic and Information Engineering, Jiujiang University, China. His research interests include signal processing, IoTs and control engineering.

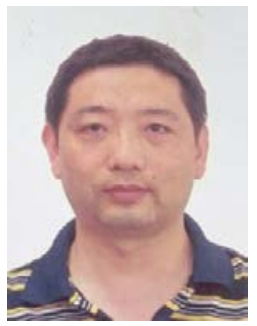

Qin Xu was born in Jiujiang, P.R. China, in 1972. He received the bachelor's degree from Nanchang Hangkong University, P.R. China. He is currently an associate Professor in the School of Electronic and Information Engineering, Jiujiang University, China. His research interests include Internet of things technology, image processing technology and big data analysis.

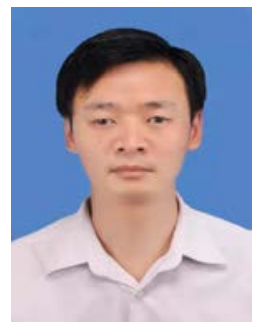

Yubao Gao was born in 1978. He received the Ph.D from Sun Yat-Sen University, Zhongshan, China, in 2015. He is currently an associate Professor in the School of Electronic and Information Engineering, Jiujiang University, China. His research interests include biomedical informatics, medical electronics. 